\title{
Enforced expression of RASAL1 suppresses cell proliferation and the transformation ability of gastric cancer cells
}

\author{
FENGCHANG QIAO $^{1 *}$, XIANWEI SU ${ }^{1 *}$, XUEMEI QIU $^{1}$, DANWEN QIAN $^{1}$, \\ XIAO PENG ${ }^{1}$, HONG CHEN $^{2}$, ZHUJIANG ZHAO ${ }^{1}$ and HONG FAN ${ }^{1}$ \\ ${ }^{1}$ Key Laboratory of Developmental Genes and Human Diseases, Ministry of Education, \\ Institute of Life Science, and Department of Genetics and Developmental Biology, \\ ${ }^{2}$ Zhong Da Hospital, Medical School of Southeast University, Nanjing 210009, P.R. China
}

Received April 26, 2012; Accepted June 19, 2012

DOI: $10.3892 /$ or.2012.1920

\begin{abstract}
RAS protein activator like 1 (RASAL1) is a member of the RAS GTPase-activating protein (GAP) family, and it is an important molecule in the regulation of RAS activation. In the present study, we investigated the role of RASAL1 in gastric carcinogenesis. Decreased expression pattern of RASAL1 in gastric cancer tissues and cell lines was found in protein and RNA levels, although there was no statistically significant relationship between RASAL1 and clinicopathological features. Restored expression of RASAL1 induced by DNA methylation inhibitor 5-aza-2'-deoxycytidine (5'-AZA) and HDAC inhibitor trichostatin A (TSA) implied that RASAL1 expression is regulated by epigenetic mechanisms. The biological role of RASAL1 in gastric carcinogenesis was determined by in vitro tumorigenicity assays. Overexpression of RASAL1 showed suppression of cell proliferation due to cell apoptosis. Subsequently, enforced expression of RASAL1 repressed significantly the gastric cancer cell transformation ability. These findings demonstrated that decreased RASAL1 expression is a characteristic of gastric cancer and regulated by epigenetic mechanisms. RASAL1 may be a functional tumor suppressor involved in gastric cancer. This study provides novel insights into the biological role of RASAL1 in gastric carcinogenesis.
\end{abstract}

\section{Introduction}

RAS proteins are molecular switches for signaling cascades that modulate many aspects of cell biology $(1,2)$. They have two distinct conformations, including an inactive GDP-bound

Correspondence to: Dr Hong Fan, Key Laboratory of Developmental Genes and Human Diseases, Ministry of Education and Department of Genetics and Developmental Biology, Medical School of Southeast University, Nanjing 210009, P.R. China

E-mail: fanh@seu.edu.cn

${ }^{*}$ Contributed equally

Key words: RASAL1, gastric cancer, cell growth, cell apoptosis form and an active GTP-bound form, which are regulated by RAS guanine nucleotide exchange factors (GEFs) and RAS GTPase-activating proteins (GAPs) (3). Approximately $30 \%$ of human tumors express an oncogenic form of RAS (i.e., Ha-, K-, or N-RAS), which, in its active form, is insensitive to Ras GAPs (3). RASAL1, which is a member of the Ras GAPs family, has been shown to be downregulated in several solid tumors, including brain, skin, bladder, head, neck, lung, liver, esophageal, and multiple cell lines. Loss of RASAL1 activity has been correlated with hyperactive Ras in the colon and hepatocellular carcinoma lacking oncogenic Ras (4,5). Matkar et al have reported that systemic $K$-ras activation in mice leads to rapid changes in gastric cellular homeostasis, and conditional $K$-ras activation results in MAPK pathway activation and the hyperproliferation of the squamous epithelium in the forestomach and metaplasia in the glandular stomach. Parietal cells almost completely disappear from the upper portion of the stomach that is adjacent to forestomach in $K$-ras activated mice (6). The activated embryonic oncogene ERas may be associated with the tumorigenic growth of somatic cells and may be a putative molecule responsible for cancer stem cell-like characteristics in gastric cancer (7). Liu et al (8) found that different types of $K$-ras mutations may play a role in gastric cancer development at different stages in the Chinese population. However, Ras mutations are rare, and normally, wild-type Ras is found in gastric cancer. Given that Ras GAPs including RASAL1 suppression may lead to aberrant Ras activation, which promotes tumorigenesis (9), in this study, we investigated RASAL1 expression patterns in gastric cancer tissues and cell lines, exploring the potential epigenetic mechanism of RASAL1 inactivation in gastric cancer cell lines. Enforced expression of RASAL1 is to evaluate its biological function in gastric cancer cells. These findings may be beneficial in exploring the mechanism and treatment options for gastric cancer.

\section{Materials and methods}

Tissue samples and cell lines. Gastric cancer and adjacent non-cancerous tissue specimens were obtained from the First Hospital of Nanjing. The samples and our study were approved by the Committees for Ethical Review of Research at the first hospital of Nanjing in China and the patients signed informed 
consent forms. The clinicopathological features are shown in Table I. Four human gastric cancer cell lines AGS, MCG-803, SGC-7901 and BGC-823 were obtained from the Chinese Academy of Science cell bank.

Western blot analysis. Western blot analysis was performed to detect RASAL1 protein expression in gastric cancer specimens and cell lines. The protein concentration of each extract was standardized using the BCA assay (Pierce, USA). The RASAL1 primary antibody (1:1500, Abcam) and the mouse monoclonal anti- $\beta$-actin antibody (1:8000, Sigma, USA) were used to detect RASAL1 protein levels. The intensities of specific protein bands were quantified with Gel Pro 3.2 (UVP, CLL, USA), corrected for the intensity of the respective $\beta$-actin band.

Reverse-transcription (RT)-PCR and quantitative real-time polymerase chain reaction ( $q P C R)$. Total RNA from the cases and cell lines was isolated using TRIzol reagent (Invitrogen, USA), and first-strand cDNA was prepared from the total RNA with an oligo $(\mathrm{dT})_{18}$ primer and AMV reverse transcriptase (BioFlux, Japan) according to the manufacturer's instructions. Rasall gene expression was examined using a SYBR-Green PCR kit (Takara, Japan). The Rasall gene RT-PCR and qPCR primers, resulting in a 265-bp DNA product, were as follows: 5'-GCAGGGAGGCGATTACAGCCGACCCCCGAG-3' (sense) and 5'-GGGAAGCGAGTCTTCTTGATGGTTGAGGTC TCC-3' (antisense) (10).

5'-AZA and TSA treatment. A total of $1.5 \times 10^{5}$ AGS, BGC-823, MCG-803 and SGC-7901 cells were plated in 6-well plates. The cells were cultured in medium containing $0,5,10$ or $50 \mu \mathrm{mol} / 1$ of the DNA methyltransferase inhibitor 5'-AZA (Sigma) for 72 h. BGC-823 cells were also treated with $0,0.1,0.2,0.3,0.4$ or $0.5 \mu \mathrm{mol} / 1 \mathrm{TSA}$ and MCG-803 cells were treated with $0,0.1$, 0.2 , or $0.3 \mu \mathrm{mol} / 1 \mathrm{TSA}$ (Sigma), a histone deacetylase inhibitor, for $24 \mathrm{~h}$.

Transfection of RASAL1 into BGC-823 and MCG-803 cells. BGC-823 and MCG-803 cells were transfected with a RASAL1 construct or pEGFP-N1 as a control (a gift from Q. Tao, Chinese University of Hong Kong) using the FuGene HD transfection reagent (Roche, Switzerland) according to the manufacturer's instructions. The cells were grown and selectively cultured in $0.4 \mathrm{mg} / \mathrm{ml} \mathrm{G} 418$ (Life Technologies, USA) for 2 months after the initial transfection. BGC-823 and MCG-803 RASAL1-transfected cells were named BGC-RASAL1 and MCG-RASAL1, respectively, and those transfected with pEGFP-N1 were referred to as the control.

Cell growth and apoptosis assay. The Cell Counting kit- 8 (CCK-8) (Dojindo Laboratories, Kumamoto, Japan) was used to measure the cellular growth of BGC-RASAL1, MCG-RASAL1 and the control cells. The 450-nm absorbance was measured to determine the cell viability. All of the experiments were independently repeated at least three times. Apoptosis was detected by PI and Annexin V-FITC staining. The cells were seeded at $3 \times 10^{5}$ cells/well in 6 -well plates and incubated for $24 \mathrm{~h}$ and $48 \mathrm{~h}$. Trypsinized cells were washed three times with PBS. The cells were then conjugated with Annexin V-FITC using the PI/Annexin V-FITC kit
Table I. The association of RASAL1 expression with clinicopathological features in $50 \mathrm{GC}$ cases.

\begin{tabular}{|c|c|c|c|}
\hline Parameters & $\mathrm{T}<\mathrm{N}$ & $\mathrm{T} \geq \mathrm{N}$ & P-value \\
\hline Age & & & 1 \\
\hline$\leq 60$ & 12 & 8 & \\
\hline$>60$ & 18 & 12 & \\
\hline Gender & & & 0.626 \\
\hline Male & 19 & 14 & \\
\hline Female & 11 & 6 & \\
\hline Differentiation & & & 1 \\
\hline Poor & 16 & 11 & \\
\hline Moderate & 13 & 9 & \\
\hline Invasive degree & & & 0.936 \\
\hline Early stage & 3 & 2 & \\
\hline Progression & 25 & 18 & \\
\hline Lymph node metastasis & & & 0.742 \\
\hline Yes & 19 & 14 & \\
\hline No & 10 & 6 & \\
\hline
\end{tabular}

T, tumor tissues; N, peri-carcinoma normal tissues.

(Biouniquer, USA), according to the manufacturer's protocol, and analyzed by flow cytometry (Olympus, Japan).

In vitro tumorigenicity assays and migration detection. For soft agar colony formation, $1 \times 10^{3}$ cells were seeded in 6-well plates with RPMI-1640 containing 10\% FBS and incubated at $37^{\circ} \mathrm{C}$ in $5 \% \mathrm{CO}_{2}$. Colonies consisting of $>80$ cells were counted after 2 weeks, and the data are expressed as the mean \pm SD. of triplicate wells within the same experiment. The foci formation assay was performed by seeding $1 \times 10^{3}$ cells in a 6 -well plate. After 12 days, the surviving colonies (450 cells per colony) were counted following crystal violet (Invitrogen) staining. Triplicate independent experiments were performed.

Cell migration was assessed by measuring the movement of cells into a scraped area created by a $200-\mu l$ pipette tip, and the spread of the wound closure was observed after $36 \mathrm{~h}$. The cells were photographed under a microscope.

Statistical analysis. Correlations between the RASAL1 expression levels and pathological features were analyzed with the $\chi^{2}$ test using SPSS 13.0 software for Windows. Differences were analyzed by Fisher's exact test. The independent Student's t-test was used to compare the results, which were expressed as the mean \pm SD between any two preselected groups. Results with a $\mathrm{P}<0.05$ were considered statistically significant.

\section{Results}

Decreased RASAL1 expression in gastric cancer cases and cell lines. The RASAL1 protein levels were detected by western blotting in 50 pairs of tumor and matched non-tumor tissue 
A
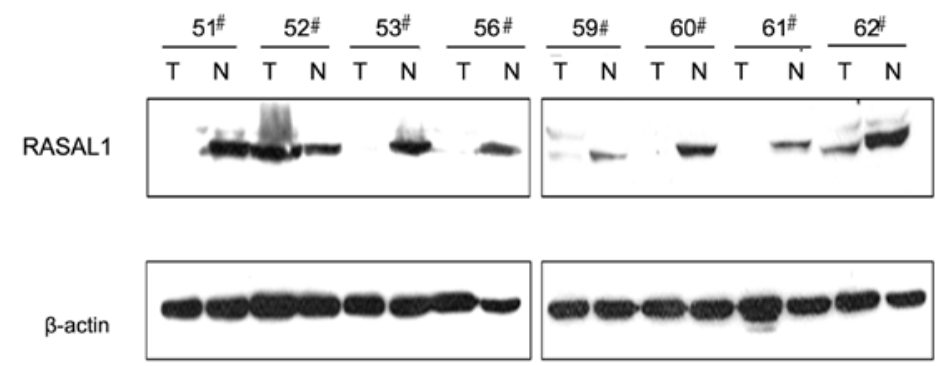

B

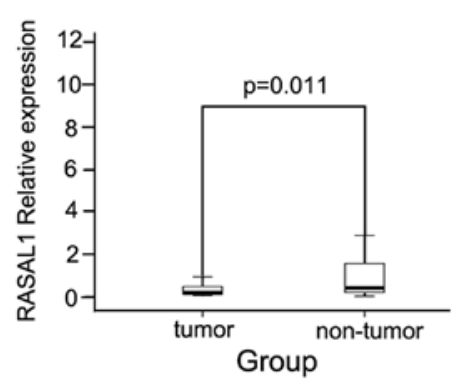

C

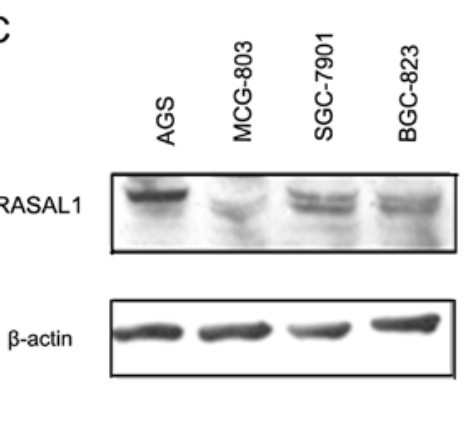

D

RASAL1 expression in GC cell lines

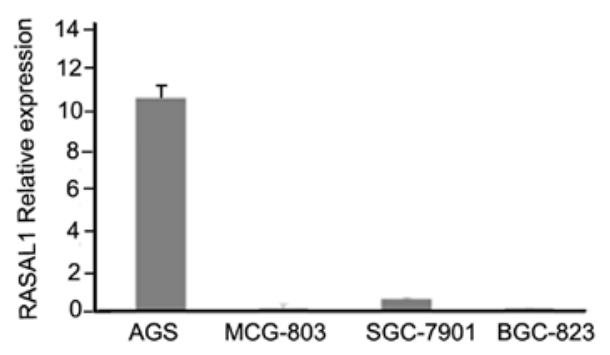

Figure 1. RASAL1 is downregulated in gastric cancer (GC) tissues and three GC cell lines compared to AGS. (A and B) RASAL1 expression level was analyzed in gastric tissues by western blotting and qPCR, respectively. $\beta$-actin was used as an internal control. (C and D) RASAL1 expression level was analyzed in GC cell lines by western blotting and qPCR, respectively. $\beta$-actin was used as an internal control.

specimens. The frequency of RASAL1 downregulation was $64 \%$ (32/50) (Fig. 1A). As detected by qPCR, the RASAL1 mRNA expression level was $58.8 \%$ (20/34), which is consistent with the protein expression level (Fig. 1B). Western blotting and qPCR demonstrated that RASAL1 expression was reduced markedly in gastric cancer cells BGC-823, 7901 and MCG-803 compared to AGS (Fig. 1C and 1D). These data indicated that decreased RASAL1 expression may play an important role in gastric tumorigenesis.

Clinicopathology of decreased RASAL1 expression in gastric cancer. To explore the clinicopathological significance of the RASAL1 expression pattern in gastric cancer tumorigenesis, the clinical features of patients with GCs were analyzed. A total of 34 patients with gastric cancer were categorized into two groups according to their RASAL1 expression level. However, there was no statistically significant association between RASAL1 and clinicopathological features, including age, gender, differentiation, invasion degree and lymph node metastasis (Table I).
Correlation between RASAL1 expression and environmental factors (H. pylori and Epstein-Barr virus infection). The presence of $H$.pylori and EB virus infection was determined by PCR for $H$. pylori $16 \mathrm{~S}$ rDNA and the EBNA-1 region, respectively, as previously described (11). The relationships between the H. pylori and Epstein-Barr virus (EBV) infection status of the patient and the RASAL1 expression level were analyzed. Of the $50 \mathrm{GC}$ cases, 22 samples were EBV-positive, and 28 samples were EBV-negative; 22 samples were $H$. pylori-positive, and 28 samples were $H$. pylori-negative (Table II). No significant correlation between the RASAL1 expression and environmental factors was observed.

Silenced RASAL1 was restored by either 5'-AZA or TSA depending on the drug concentration. To investigate whether the RASAL1 downregulation observed in gastric cancer tissues and cell lines is due to epigenetic inactivation, gastric cancer cell lines AGS, BGC-823, MCG-803 and SGC-7901 were treated with DNA methylation inhibitor 5-aza-2'-deoxycytidine (5'-AZA) or HDAC inhibitor trichostatin A (TSA). RASAL1 expression was 
Table II. Association of RASAL1 expression with H. pylori and $\mathrm{EBV}$ infection in $50 \mathrm{GC}$ cases.

\begin{tabular}{lrrrr}
\hline & Cases & $\mathrm{T}<\mathrm{N}$ & $\mathrm{T} \geq \mathrm{N}$ & P-value \\
\hline $\begin{array}{l}\text { H. pylori and EBV infection } \\
\text { Both }\end{array}$ & 9 & 5 & 4 & \\
Either & 26 & 15 & 11 & \\
Neither & 15 & 11 & 4 & \\
H. pylori or EBV infection & & & & 0.554 \\
$\quad(+)$ & 35 & 20 & 15 & \\
$(-)$ & 15 & 11 & 4 & \\
\hline
\end{tabular}

T, tumor tissues; N, peri-carcinoma normal tissues; (+), positive; (-), negative.

restored after treatment with 5'-AZA for $72 \mathrm{~h}$ in the three gastric cell lines, depending on the drug concentration (MCG-803 and BGC-823: 0-50 $\mu \mathrm{mol} / 1$; SGC-7901: 5-50 $\mu \mathrm{mol} / \mathrm{l}$ ) (Fig. 2A). Moreover, RASAL1 expression was also restored depending on the TSA concentration in the BGC-823 (0-0.5 $\mu \mathrm{mol} / \mathrm{l})$ and MCG-803 (0-0.3 $\mu \mathrm{mol} / \mathrm{l})$ cell lines (Fig. 2B). These findings demonstrate the expression of RASAL1 is affected by epigenetic modification in gastric cancer cells.

Restoration of RASAL1 expression inhibited tumor cell proliferation by inducing apoptosis. BGC-823 and MCG- 803 cells were stably transfected with a pEGFP-RASAL1 expression construct or a pEGFP control construct. RASAL1 expression increased in the RASAL1-transfected cells (BGC-RASAL1 \#5 and \#6 and MCG-RASAL1 \#2) as compared with the pEGFP-transfectants. RASAL1 overexpression decreased the cell growth rates of the BGC-RASAL1 \#5 and 6 cells as compared with the BGC-pEGFP control, especially from the third to the fifth day (Fig. 3A). The effect of RASAL1 overexpression on cell growth in the MCG-RASAL1 \#2 cells was more significant beginning on the second day (Fig. 3B). Flow cytometric analysis showed that RASAL1 overexpression increased apoptosis in the BGC-RASAL1 \#6 cells at $48 \mathrm{~h}$. (15.7 vs $9.29 \%,{ }^{*} \mathrm{P}=0.041$; Fig. 3C). The MCG-RASAL1 \#2 cells showed significantly induced apoptosis as compared to the MCG-pEGFP control at $24 \mathrm{~h}\left(15.48\right.$ vs $\left.12.18 \%,{ }^{*} \mathrm{P}=0.026\right)$ and $48 \mathrm{~h}$ (19.45 vs. $\left.14.54 \%,{ }^{*} \mathrm{P}=0.035\right)$ (Fig. 3D). These data implied that RASAL1 inhibited gastric cancer cell line growth by inducing cell apoptosis.

Enforced expression of RASAL1 suppressed gastric cancer cells transformation and migration ability. RASAL1 tumor-
A
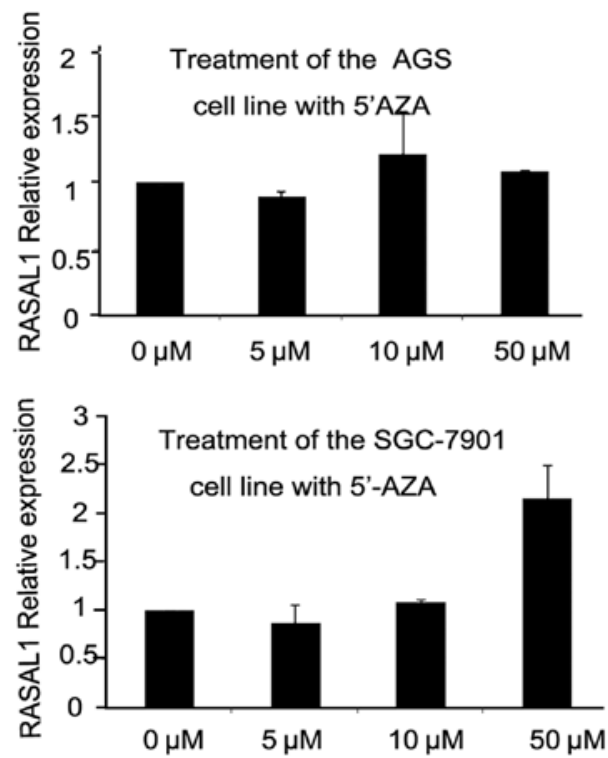

B

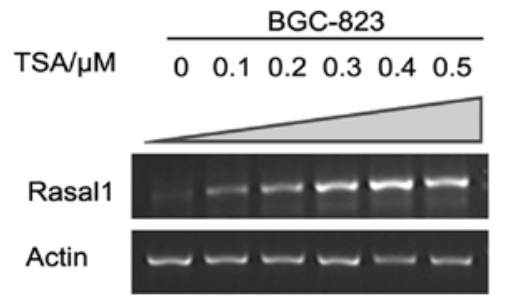

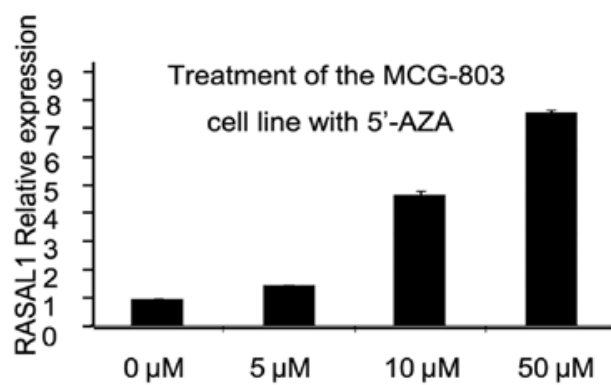
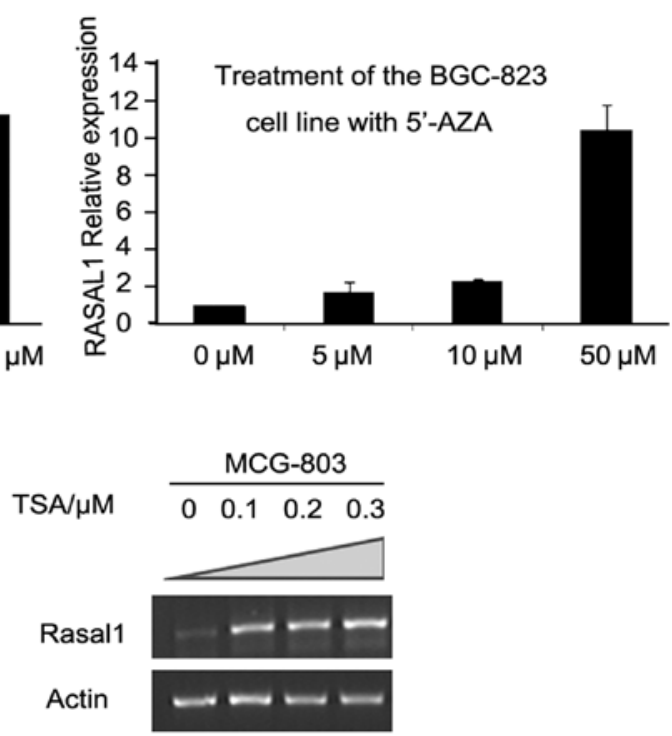

Figure 2. RASAL1 expression was restored by epigenetic modification drugs. (A) RASAL1 expression was detected in gastric cancer cell lines (AGS, MCG-803, SGC-7901 and BGC-823) after 5'-AZA treatment $(0,5,10$ and $50 \mu \mathrm{M})$ for $72 \mathrm{~h}$ by qPCR. (B) RT-PCR showed RASAL1 expression after induced for $24 \mathrm{~h}$ by $0,0.1$, $0.2,0.3,0.4$ and $0.5 \mu \mathrm{M}$ TSA in the BGC-823 cell line and by $0,0.1,0.2$ and $0.3 \mu \mathrm{M}$ in the MCG-803 cell line, respectively. 
A
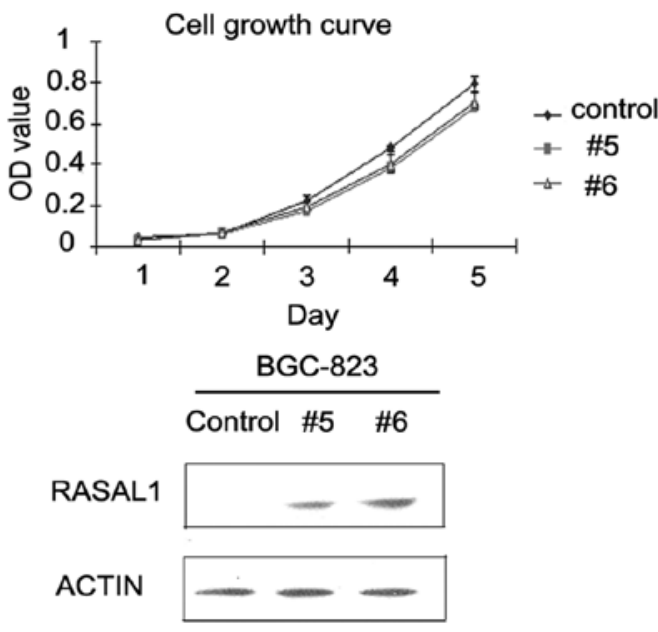

C
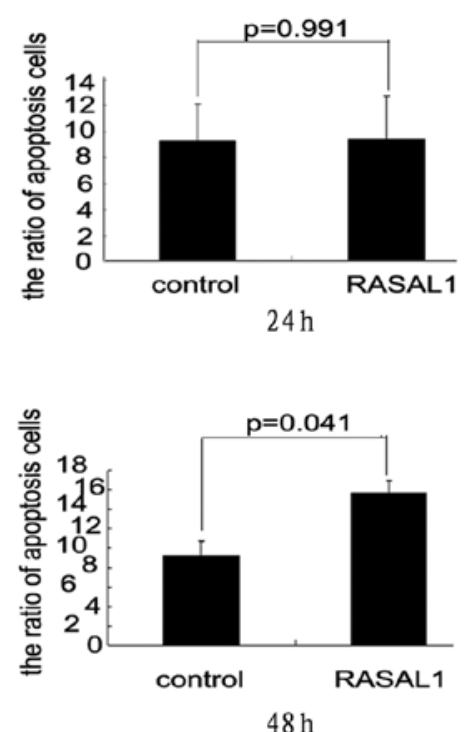

B
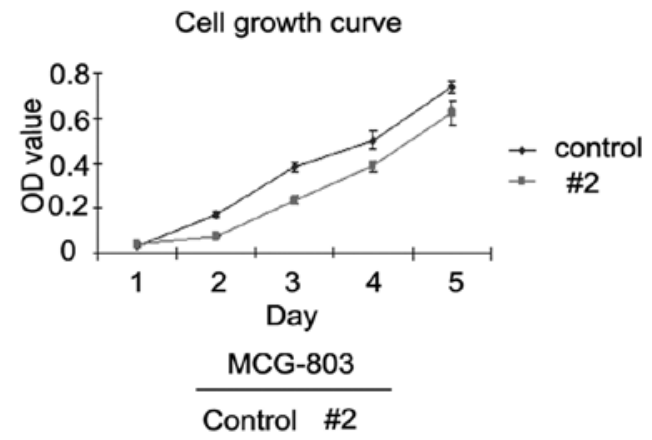

RASAL1

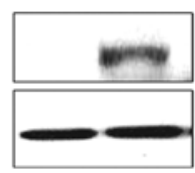

ACTIN
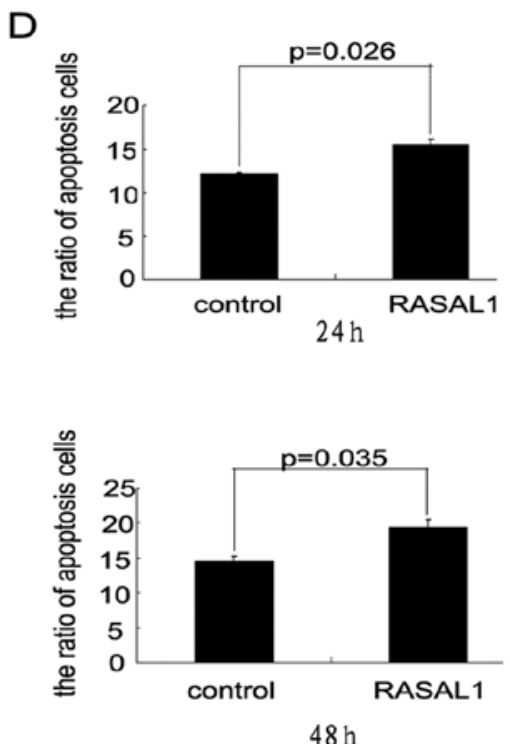

Figure 3. Overexpression of RASAL1 inhibited cell growth by induction of apoptosis. (A and B) Inhibition of cell growth were detected by CCK-8 in BGC-823 and MCG-803 cells which were stably transfected with RASAL1 construct. (C and D) Apoptosis rate was evaluated by FACS at 24 h and 48 h in BGC-823 cells and MCG-803 cells with RASAL1 enforced expression.

suppressive effects were assessed by colony and foci formation assays in soft agar and wound healing assays. The frequency of foci formation was significantly lower in the BGC-RASAL1 \#6 cells compared to the BGC-pEGFP control cells $(\mathrm{P}=0.004$; Fig. $4 \mathrm{~A})$. Colony formation in soft agar was significantly decreased in the BGC-RASAL1 \#6 cells compared to the BGC-pEGFP cells $(\mathrm{P}=0.000$; Fig. 4B). Additionally, wound healing assay showed that RASAL1 inhibited cell migration ability in BGC cells (Fig. 4C). Together, these data provide evidence that RASAL1 has significant tumor-suppressive effects in gastric cancer cells.

\section{Discussion}

Ras GAPs play an important role in regulating Ras activation, which is an alternative mechanism to Ras gene mutation. RASAL1 is a member of the Ras GAPs family, and it is a $\mathrm{Ca}^{2+}$-regulated Ras GAPs that decodes the frequency of $\mathrm{Ca}^{2+}$ oscillations (10). Other family members include p120GAP, neurofibromin (NF1), the GAP1 family (GAP1 ${ }^{\mathrm{IP} 4 \mathrm{BP}}, \mathrm{Ca}^{2+}-$ promoted Ras inactivator (CAPRI), and Ras GTPase activating-like protein RASAL1), and the SynGAP family (DAB2IP, nGAP, SynGAP) (9,12-18). The expression profiles and cellular localizations of the Ras GAP family members differ. Previous studies have shown a reduction in RASAL1 expression in nasopharyngeal carcinoma, breast, lung, liver, and esophageal squamous cell carcinoma cell lines (10) as well as in colon (5).

Our findings showed that RASAL1 is downregulated in gastric cancer tissues. Although most gastric cancers are considered to be derived from chronic inflammatory mucosa induced by $H$. pylori and Epstein-Barr virus (EBV) infection, decreased expression of RASAL1 was not observed relationships between the RASAL1 expression level and H.pylori and Epstein-Barr virus (EBV) infection, Seto et al (19) also reported that reduced expression of RASAL1 was not observed in any inflammatory mucosa or intestinal metaplasia. Therefore, RASAL1 possibly contributes to gastric carcinogenesis as a tumor suppressor gene (TSG), but is not effected by environment factors in gastric 
A

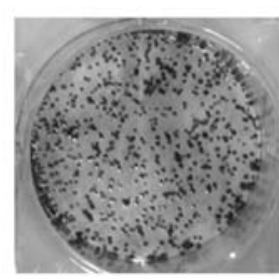

control
BGC-823

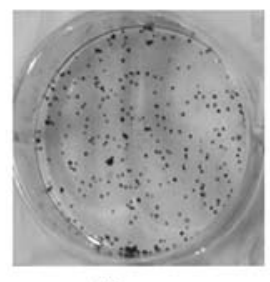

\#6

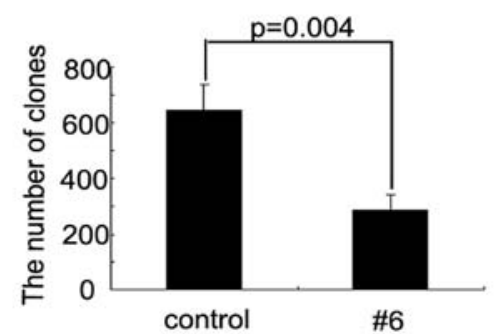

C

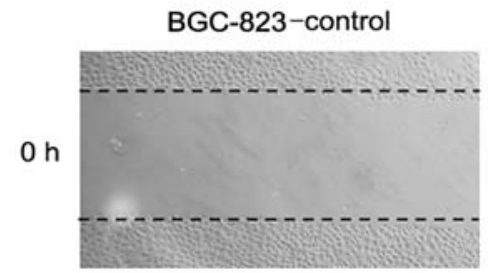

$36 \mathrm{~h}$

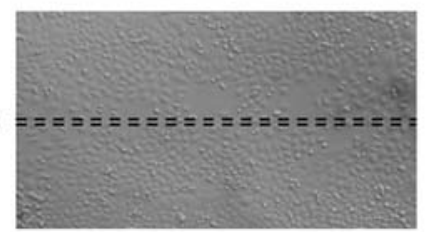

B

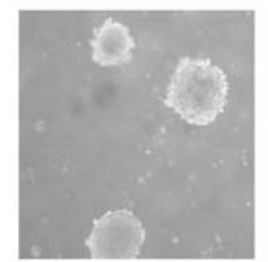

control
BGC-823

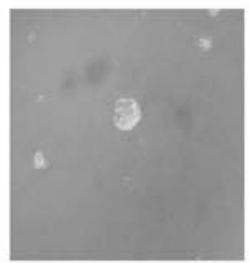

\#6

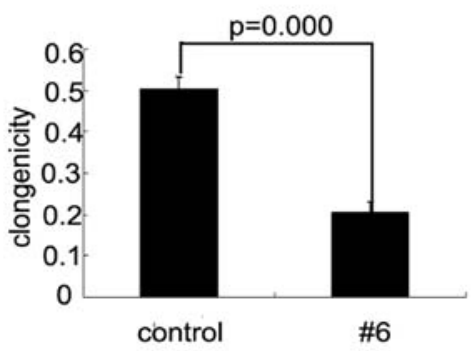

BGC-823-\#6
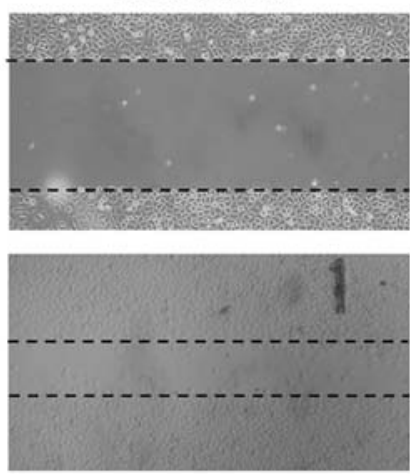

Figure 4. RASAL1 overexpression shows its tumor suppressor effect in vitro tumorigenicity assays. (A and B) RASAL1 reduced the cell colony and foci formation abilities in BGC-823 cell line. Quantitative analyses of the colony and foci numbers are shown as the mean \pm SD. (C) RASAL1 overexpression inhibited cell migration in wound healing assays of the BGC-823 cell line at $36 \mathrm{~h}$.

cancer. However, the potential role of RASAL1 in gastric progression and its ability to inhibit gastric tumorigenicity is less well known. In order to explore the biological role of RASAL1 in gastric tumorigenesis, we established RASAL1 overexpression cell models and evaluated whether RASAL1 restoration affects the malignant phenotype of tumor cells. The present study found that RASAL1 overexpression inhibited cell proliferation induced by cell apoptosis. RASAL1 enforced expression significantly inhibited foci formation and cell migration, which implied RASAL1 is associated with the epithelial-mesenchymal transition (EMT) process, and EMT-related markers should be identified.

A previous report has found that RASAL1 downregulation resulted from an underexpressed transcription factor PITX1 in certain tumors (20). However, decreased expression of RASAL1 was not observed related with the expression of PITX1 (data not shown) in our study. Jin et al (10) have reported an epigenetic silencing mechanism of RASAL1 in a variety of carcinomas. In this study, silenced RASAL1 was restored after treatment with 5'-AZA or TSA depending on the drug dose. Together with previous studies, our findings supported that DNA methylation and histone deacetylation contribute, at least in part, to reduced RASAL1 expression in gastric carcinogenesis.
In conclusion, the present study provide the first insight into the biological function of RASAL1 and implied its potential role in gastric progression and its ability to inhibit gastric tumorigenicity. Our findings are useful in understanding if RASAL1 may be beneficial for the development of new treatment options for gastric cancer. However, the underlying molecular mechanism by which RASAL1 promotes apoptosis and controls gastric carcinogenesis remains to be determined.

\section{Acknowledgements}

This study was supported by the National Natural Science Foundation of China, grant no. 81171915. This study was also supported by Nanjing Medical Key Scientific Foundation, (no. ZKX08012). We are grateful to Professor Qian Tao for providing the pEGFP-RASAL1 plasmid.

\section{References}

1. Hancock JF: Ras proteins: different signals from different locations. Nat Rev Mol Cell Biol 4: 373-384, 2003.

2. Mitin N, Rossman KL and Der CJ: Signaling interplay in Ras superfamily function. Curr Biol 15: R563-R574, 2005. 
3. Downward J: Role of receptor tyrosine kinases in G-proteincoupled receptor regulation of Ras: transactivation or parallel pathways? Biochem J 376: e9-10, 2003.

4. Calvisi DF, Ladu S, Conner EA, et al: Inactivation of Ras GTPaseactivating proteins promotes unrestrained activity of wild-type Ras in human liver cancer. J Hepatol 54: 311-319, 2011.

5. Ohta M, Seto M, Ijichi H, et al: Decreased expression of the RAS-GTPase activating protein RASAL1 is associated with colorectal tumor progression. Gastroenterology 136: 206-216, 2009.

6. Matkar SS, Durham A, Brice A, Wang TC, Rustgi AK and Hua X: Systemic activation of K-ras rapidly induces gastric hyperplasia and metaplasia in mice. Am J Cancer Res 1: 432-445, 2011.

7. Yashiro M, Yasuda K, Nishii T, et al: Epigenetic regulation of the embryonic oncogene ERas in gastric cancer cells. Int J Oncol 35: 997-1003, 2009.

8. Liu ZM, Liu LN, Li M, Zhang QP, Cheng SH and Lu S: Mutation detection of KRAS by high-resolution melting analysis in Chinese with gastric cancer. Oncol Rep 22: 515-520, 2009.

9. Bernards A and Settleman J: GAPs in growth factor signalling. Growth Factors 23: 143-149, 2005.

10. Jin H, Wang X, Ying J, et al: Epigenetic silencing of a $\mathrm{Ca}(2+)-$ regulated Ras GTPase-activating protein RASAL defines a new mechanism of Ras activation in human cancers. Proc Natl Acad Sci USA 104: 12353-12358, 2007.

11. Su X, Lv C, Qiao F, et al: Expression pattern and clinical significance of DNA methyltransferase 3B variants in gastric carcinoma. Oncol Rep 23: 819-826, 2010
12. Yarwood S, Bouyoucef-Cherchalli D, Cullen PJ and Kupzig S: The GAP1 family of GTPase-activating proteins: spatial and temporal regulators of small GTPase signalling. Biochem Soc Trans 34: 846-850, 2006.

13. Cullen PJ and Lockyer PJ: Integration of calcium and Ras signalling. Nat Rev Mol Cell Biol 3: 339-348, 2002.

14. Donovan S, Shannon KM and Bollag G: GTPase activating proteins: critical regulators of intracellular signaling. Biochim Biophys Acta 1602: 23-45, 2002.

15. Le LQ and Parada LF: Tumor microenvironment and neurofibromatosis type I: connecting the GAPs. Oncogene 26: 4609-4616, 2007.

16. Dasgupta B and Gutmann DH: Neurofibromatosis 1: closing the GAP between mice and men. Curr Opin Genet Dev 13: 20-27, 2003.

17. Pamonsinlapatham P, Hadj-Slimane R, Lepelletier Y, et al: p120-Ras GTPase activating protein (RasGAP): a multi-interacting protein in downstream signaling. Biochimie 91: 320-328, 2009.

18. Grewal T and Enrich C: Molecular mechanisms involved in Ras inactivation: the annexin A6-p120GAP complex. Bioessays 28: 1211-1220, 2006.

19. Seto M, Ohta M, Ikenoue T, et al: Reduced expression of RAS protein activator like-1 in gastric cancer. Int J Cancer 128: 1293-1302, 2011.

20. Kolfschoten IG, van Leeuwen B, Berns K, et al: A genetic screen identifies PITX1 as a suppressor of RAS activity and tumorigenicity. Cell 121: 849-858, 2005. 